\title{
Patch Angioplasty for a Left Main Coronary Artery Pseudoaneurysm following Percutaneous Coronary Intervention
}

\author{
Keigo Yamashita, Takehisa Abe, Nobuoki Tabayashi, Yoshihiro Hayata, \\ Tomoaki Hirose, Shun Hiraga, Shigeki Taniguchi
}

\begin{abstract}
A left main coronary artery pseudoaneurysm is rare and the treatment strategies are poorly defined. Herein we report a surgical treatment for a left main coronary artery pseudoaneurysm in a 56-year-old female patient with end-stage renal disease on hemodialysis. Patch angioplasty with a saphenous vein graft was performed for the left main coronary artery pseudoaneurysm following percutaneous coronary intervention with a rotablator. She underwent a living renal transplantation one year after surgery and then has been doing well these 2 years.

KEY WORDS: patch angioplasty, left main coronary artery, coronary artery pseudoaneurysm, percutaneous coronary intervention
\end{abstract}

\section{Text}

A coronary artery pseudoaneurysm usually occurs following catheter-based intervention, probably due to traumatic dissection or perforation of a coronary artery resulting in the disruption of the media without blood seepage through the adventitia. ${ }^{1} \mathrm{Be}-$ cause of rarity of this condition, treatment strategies are not clearly defined. Herein we report a patient who presented with a left main coronary artery pseudoaneurysm.

A 56-year-old woman, who had been on hemodialysis for end-stage renal disease due to chronic glomerulonephritis 5 years before, was referred to our hospital because she had a saccular aneurysm in the left main coronary artery. She had percutaneous coronary intervention (PCI) to the diagonal branch using a rotablator at the age of 54 (Fig. 1A, B). The aneurysm was diagnosed with a pseudoaneurysm by intravascular ultrasound (IVUS), the entry of which was located in the left main coronary artery just proximal to its bifurcation (Fig. 2A, B). Then the pseudoaneurysm was indicated for surgical intervention. Preoperative contrast computed tomography showed the degree of calcification in the left main coronary artery (Fig. 3A, B).

After the patient had median sternotomy and systemic heparinization made, she was placed on cardiopulmonary bypass (CPB). Retrograde cold blood cardioplegia was continuously

Department of Thoracic and Cardiovascular Surgery, Nara Medical University School of Medicine, 840 Shijo-cho, Kashihara city, Nara 634-8522, Japan

Received January 28, 2014; Accepted May 14, 2014

doi: 10.7793/jcoron. 20.14-00003 administered for myocardial protection. The pulmonary artery was transected $2 \mathrm{~cm}$ proximal to its bifurcation to optimize the exposure. An aneurysm $8 \mathrm{~mm}$ in size was found to be located in the left main coronary artery (Fig. 4A, B). The wall of the aneurysm was incised longitudinally from the left main to the proximal left anterior descending coronary artery. The damaged arterial wall was excised and repaired with a saphenous vein patch $(12 \times 25 \mathrm{~mm})$ sutured to the firm arterial wall with a 7-0 polypropylene, while the orifice of the circumflex artery was spared. There was no thrombus in the lumen. Pulmonary artery continuity was restored using a 5-0 polypropylene suture. The patient was weaned easily from CPB and her postoperative course was uneventful. The histological study of the damaged arterial wall confirmed the diagnosis of a pseudoaneurysm (Fig. 5). Postoperative contrast computed tomography showed the anastomosis site was acceptable (Fig. 6). She underwent a living renal transplantation one year postoperatively and has been doing well.

\section{Comment}

A coronary artery pseudoaneurysm is as rare a complication as a coronary artery perforation in catheter-based intervention. It is diagnosed by IVUS as a "large, thin-walled, narrow-necked, saccular, vascular structure having no normal arterial wall elements and communicating with the real arterial lumen through a rupture in the arterial wall. ${ }^{1)}$ Using IVUS, Maehara and colleagues studied 77 consecutive patients with native coronary artery aneurysms by angiography. Of these, only $27 \%$ were true aneurysms by IVUS and $4 \%$ were pseudoaneurysms. The remaining aneurysmal changes were complex plaques in $16 \%$ and 
J Jpn Coron Assoc 2014; 20: 330-333
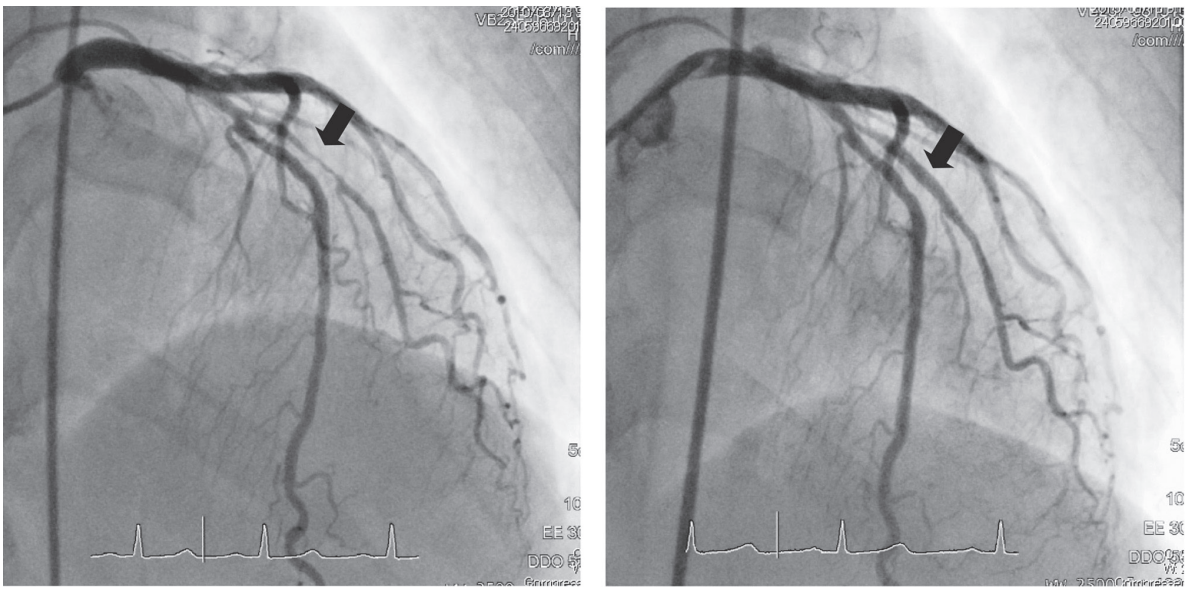

A $\mid$ B

Fig. 1

A: Left coronary arteriogram in the left anterior oblique view shows severe stenosis in the diagonal branch (arrow).

B: Final angiographic results after PCI using a rotablator (arrow).
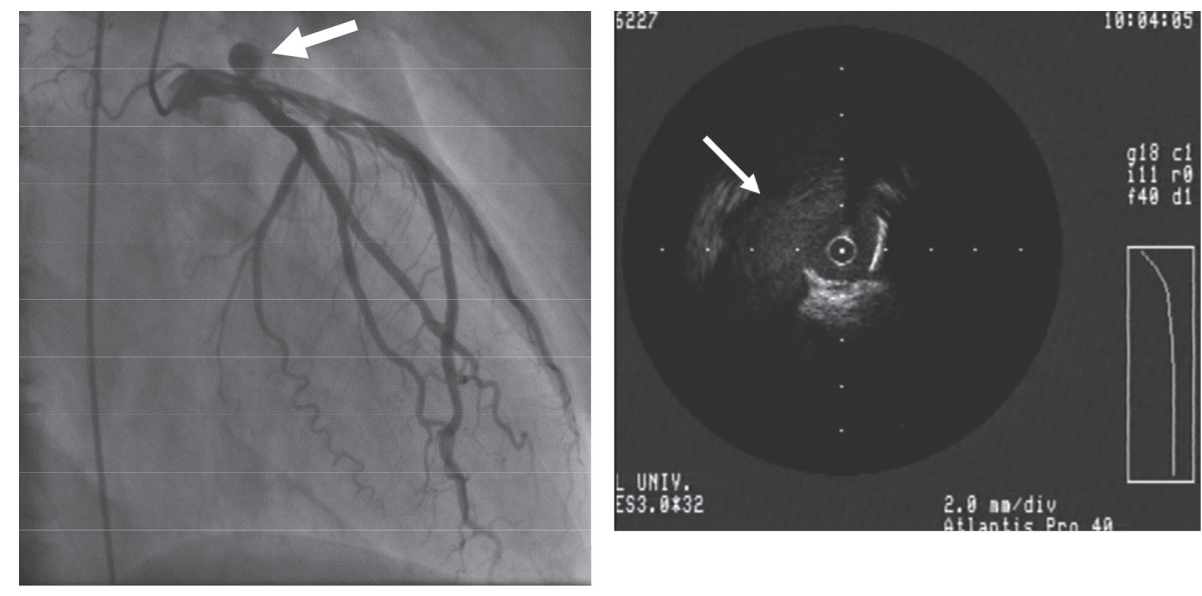

\section{Fig. 2}

A: Left coronary arteriogram in the right anterior oblique view shows a saccular aneurysm involving the left main coronary artery (arrow).

B: Intravascular ultrasound (IVUS) of a left main coronary artery pseudoaneurysm (arrow).
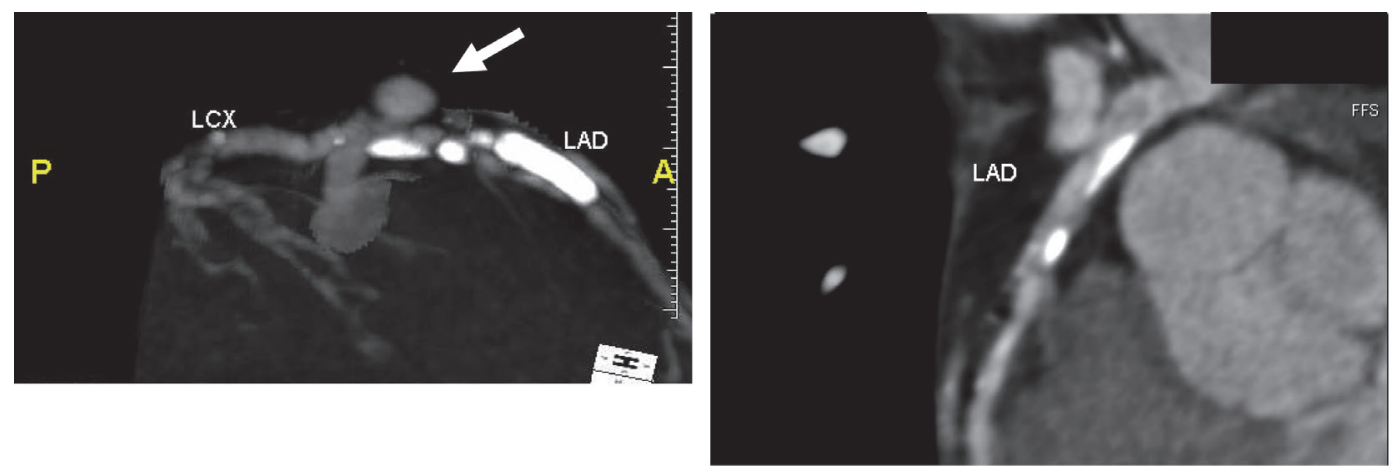

A $\mid$ B

Fig. 3 Preoperative contrast computed tomography showed the degree of calcification in the left main coronary artery. 

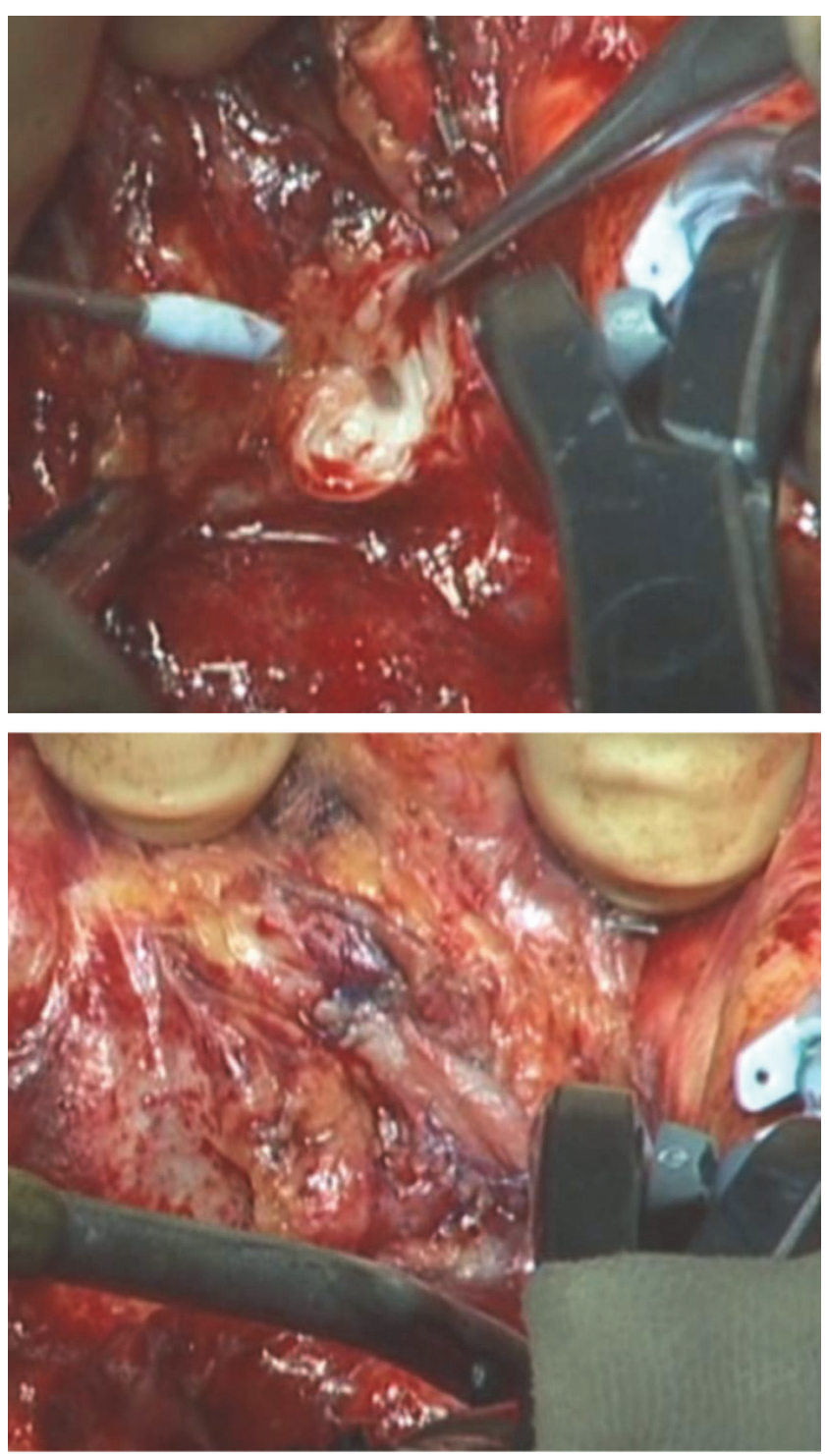

Fig. 4

A: A left main coronary artery pseudoaneurysm with the wall incised.

B: Completed patch plasty with a saphenous vein graft.

normal arterial segments adjacent to stenotic lesions in 53\%. ${ }^{2)}$ It is difficult to make a diagnosis of an aneurysm or a pseudoaneurysm accurately with coronary angiography alone. In our case, the diagnosis of a pseudoaneurysm was preoperatively made by IVUS and it was also confirmed postoperatively by pathological study.

There is no standard therapy for a coronary artery aneurysm or pseudoaneurysm. ${ }^{1)}$ Mikhail, et al. reported that some patients were left untreated and did well with a pseudoaneurysm spontaneously disappearing in one of them. ${ }^{3)}$ The indications for intervention may include the rupture or rapid enlargement of a pseudoaneurysm. This is because pseudoaneurysms have been associated with adverse outcomes, including the distal coronay em-

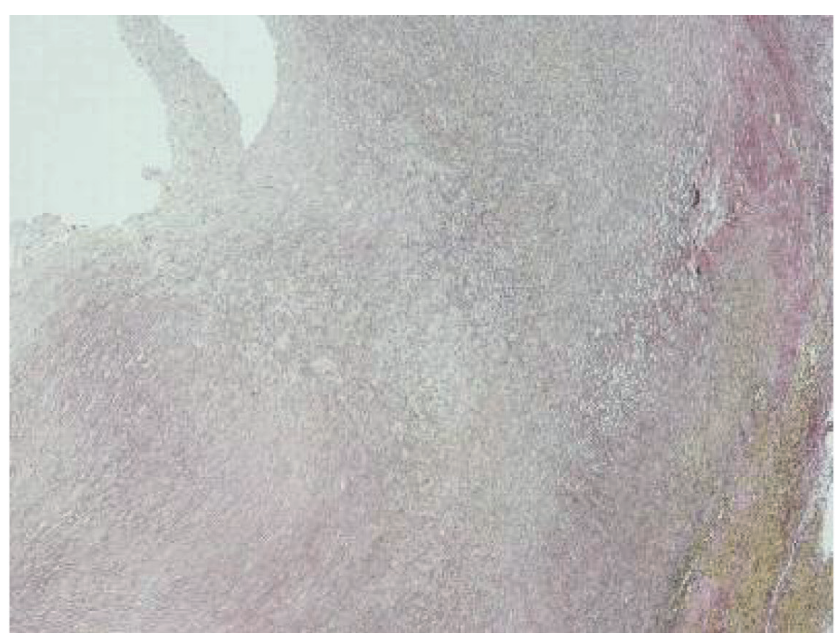

Fig. 5 Histological finding of the aneurysmal wall (Elastica van Gieson stain, $\times 40$ ).

Neither the intima or media was observed in the aneurysmal wall.

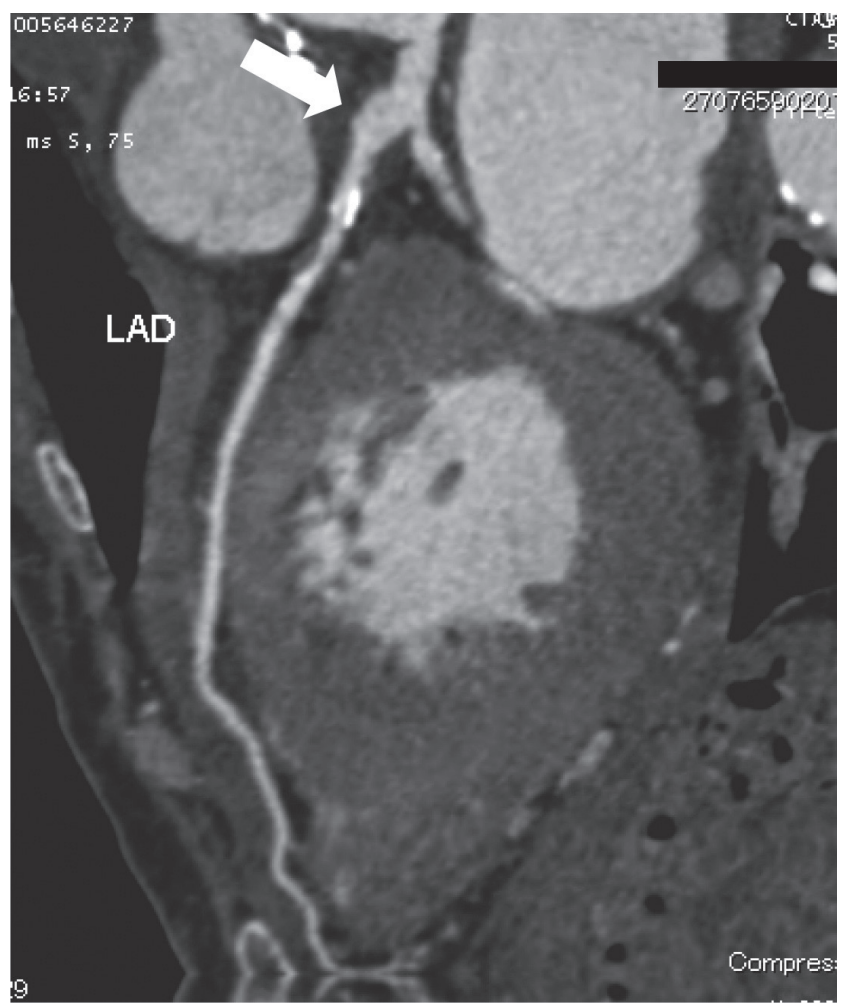

Fig. 6 Postoperative contrast computed tomography showed the anastomosis site was acceptable (arrow).

bolization and cardiac tamponade, if untreated. ${ }^{4,5)}$. Our patient was concerned about such adverse events and decided to undergo some intervention because kidney transplantation was scheduled. Dhakam, et al. reported that PCI with covered stent was used successfully to treat a coronary artery pseudoaneurysm. ${ }^{6)}$ In our case, however, PCI was unlikely to be 
recommended when we took the location of the aneurysm into consideration. The most common surgical technique may involve ligation of an aneurysm and distal bypass, which was described for the treatment of a left main coronary artery aneurysm. ${ }^{7)}$ However, the disadvantage of this strategy is that PCI cannot be performed when revascularization is necessary for a new lesion of the left coronary artery system in the future. Therefore, we chose a patch angioplasty as the surgical intervention. A saphenous vein patch is usually preferred to a fresh autologous pericardial one. ${ }^{8)}$ A fresh autologous pericardial patch may be subjected to calcification and does not have fibrinolytic properties. Autologous arterial patches have a limited width. Malyshev, et al. reported that the rate of restenotic failure in the autologous vein patch group was about 3.4\%.9) Therefore, we think that a patch angioplasty with a saphenous vein graft for a left main coronary artery pseudoaneurysm is acceptable, although we need to follow up the patency of the left main coronary artery repaired with a saphenous vein patch.

In conclusion, we demonstrated that a left main coronary artery pseudoaneurysm following PCI in a patient on hemodialysis was successfully repaired using patch angioplasty.

\section{References}

1) Aqel RA, Zoghbi GJ, Iskandrian A: Spontaneous coronary artery dissection, aneurysms, and pseudoaneurysms: a review. Echocardiography 2004; 21: 175-182

2) Maehara A, Mintz GS, Ahmed JM, et al: An intravascular ultrasound classification of angiographic coronary artery aneurysms. Am J Cardiol 2001; 88: 365-370

3) Mikhail B, Brewer RJ, Clark VL: Spontaneous closure of a perforation-induced coronary artery pseudoaneurysm. J Invasive Cardiol 2002; 14: 282-284

4) Barra JA, Bezon E, Mondine P, et al: Coronary artery reconstruction for extensive coronary disease: 108 patients and two year follow-up. Ann Thorac Surg 2000; 70: 1541-1545

5) Maehara A, Mintz GS, Castagna MT, et al: Intravascular ultrasound assessment of spontaneous coronary artery dissection. Am J Cardiol 2002; 89: 466-468

6) Dhakam S, Ahmed H, Ahmeed H, et al: Percutaneous coronary intervention of left main pseudoaneurysm with customized covered stents. Catheter Cardiovasc Interv 2011; 77: 1033-1035

7) Türkay C, Gölbaşi I, Sahin N, et al: Surgical management of an atherosclerotic aneurysm of the left main coronary artery. J Thorac Cardiovasc Surg 2001; 122: 626-627

8) Dion R, Elias B, El Khoury G, et al: Surgical angioplasty of the left main coronary artery. Eur J Cardiothorac Surg 1997; 11: 857-864

9) Malyshev M, Gladyshev I, Safuanov A, et al: Surgical angioplasty of the left main coronary artery and/or proximal segment of the right coronary artery by pulmonary autograft patch. Eur J Cardiothorac Surg 2004; 25: 21-25 